\title{
Le musée de Marine du Louvre : un musée des techniques?
}

The Musée de Marine du Louvre: A Museum of Technology?

\section{Géraldine Barron}

\section{(2) OpenEdition}

\section{Journals}

Édition électronique

URL : http://journals.openedition.org/artefact/695

DOI : $10.4000 /$ artefact.695

ISSN : 2606-9245

Éditeur :

Association Artefact. Techniques histoire et sciences humaines, Presses universitaires du Midi

Édition imprimée

Date de publication : 15 juin 2017

Pagination : 143-162

ISBN : 978-2-7535-6525-8

ISSN : 2273-0753

Référence électronique

Géraldine Barron, «Le musée de Marine du Louvre : un musée des techniques ? », Artefact [En ligne], 5 | 2016, mis en ligne le 15 novembre 2017, consulté le 08 mars 2020. URL : http://

journals.openedition.org/artefact/695; DOI : https://doi.org/10.4000/artefact.695 


\section{Le musée de Marine du Louvre : un musée des techniques?}

Géraldine BARRON*

\section{Résumé}

Le musée de Marine de Paris est constité en 1827 autour de collections de modèles réduits de navires. Le projet initial est de conserver et d'exposer la construction navale, ses outils et ses infrastructures. Mais la présence de ce musée au Louvre et son rattachement à l'administration des Beaux-Arts pendant plus de soixante-dix ans brouille l'identité de ses collections. La fonction du musée, entre lieu de conservation, outil de recherche et d'instruction et instrument de propagande, fait aussi l'objet d'interrogations récurrentes.

Mots-clés : collections, construction navale, marine, musées, techniques.

\section{Abstract. The Musée de Marine du Louvre : A Museum of Technology?}

Paris Maritime Museum was constituted in 1827 by building on the collections of model ships and it was initial to preserve and display the shipbuilding tools and infrastructure. Yet since the museum was located within the Louvre, it was administratively under the authority of the Administration of Fine Arts. Thus, for more than seventy years, the identity of its collections was undefined and its role, as preservation, research, instruction and propaganda site, was also frequently questioned.

Keywords: collections, museums, navy, shipbuilding, techniques.

\footnotetext{
* Géraldine Barron est conservatrice à la bibliothèque de l'université du Littoral Côte d'Opale. Ses recherches portent sur l'histoire maritime et l'histoire des techniques au XIX ${ }^{\mathrm{e}}$ siècle [http:/ / paris.hypotheses. org]. Dernières publications : "La corvette à vapeur l'Archimède au bout du monde, allegro ma non troppo ", Chronique d'histoire maritime, $\mathrm{n}^{\circ}$ 81, 2016, p. 67-83 et « Dévoiler la vapeur. La construction navale au XIX ${ }^{e}$ siècle », in Yves Bouvier et Léonard LABORIE (dir.), L'Europe en transitions. Énergie, mobilité, communication, XVIII -XXI siècles, Paris, Nouveau Monde éditions, 2016, p. 119-140. Contact : [gbarron76@gmail.com].
} 
L'histoire des musées de science et de technique et de leurs collections est un champ de recherche encore émergent mais dynamique, comme en témoignent les projets lancés simultanément au début de cette décennie : on citera notamment l'ouvrage récent Penser, classer, administrer : pour une histoire croisée des collections scientifiques ${ }^{1}$ ou le programme ANR-08BLAN-0071-01 «Les présents du passé » et son volet maritime, Neptune au musée ${ }^{2}$ qui s'est consacré à la mise en musée des thématiques maritimes et navales. Citons enfin le séminaire de recherche du Centre Alexandre Koyré, "Penser/ classer les collections techniques ", qui, de 2013 à 2016, a donné lieu à de nombreux échanges sur les modes de constitution des collections techniques tout en cherchant à définir une méthodologie de recherche adaptée à leur étude. les reconstitutions et les modèles, outils de médiation mais aussi de plus en plus outils de recherche. L'étude des modes de collecte, des cadres de classement, des choix de dénomination des articles, de leur mise en espace et des différents processus de valorisation éclairent non seulement l'histoire des institutions qui rassemblent ces objets scientifiques ou techniques, mais amènent aussi à repenser la place de ces objets dans la culture matérielle de différentes époques.

L'histoire du musée national de la Marine et de ses collections reste encore largement à écrire malgré les louables efforts de la revue Neptunia éditée par l'Association des amis du musée; Alain Niderlinder y a notamment reconstitué le fil chronologique de la constitution des collections ${ }^{3}$, cadre précieux pour toute étude historique. Au sein même du

musée, les sources permettant de reconstituer l'origine et la vie des collections sont assez mal répertoriées, mais un travail est heureusement en cours pour les identifier et les inventorier. Le musée conserve principalement les dossiers d'œuvres ainsi que les inventaires, les catalogues et un certain nombre de pièces d'archives. Les sources administratives sont dispersées du fait des tutelles et des localisations successives du musée. Le musée de Marine est en effet fondé au début du XIX ${ }^{\mathrm{e}}$ siècle au cœur du Louvre où il demeure jusqu'à l'ouverture des espaces actuels dans l'aile Passy du palais de Chaillot, en 1943. Il est successivement rattaché à la Maison du Roi, au ministère de la Marine et des Colonies, à l'administration des Beaux-Arts, avant son retour définitif dans le giron de la Marine et son intégration dans le périmètre du Service historique de la marine. Les archives administratives de la période qui nous intéresse ici sont principalement conservées dans les archives des musées nationaux, série EM récemment versée aux Archives nationales; on trouvera aussi quelques documents intéressants dans les archives de l'administration des Beaux-Arts du ministère de l'Instruction publique. Ces sources permettent de reconstituer les modes de collecte et de classement de ces collections de marine dont la place au cœur du Louvre suscite une longue controverse, qui cristallise l'opposition entre beauxarts et arts utiles et permet d'interroger le statut de ces collections, entre art, technique et instrument de propagande. 


\section{Les origines du musée de Marine}

Jusqu'au xviII ${ }^{\mathrm{e}}$ siècle, le maître-charpentier conçoit un navire à partir d'une vingtaine de mesures définies par l'expérience et la tradition familiale, dont la longueur, la largeur et le creux de la coque, et la construction s'effectue à partir d'un gabarit du maitre-couple. Une ordonnance de Colbert de 1679 prescrit aux intendants des arsenaux « qu'il soit fait, en chaque arsenal, des modèles en petit d'un des vaisseaux de chacun des cinq rangs ». L'obligation de fournir un plan de vaisseau est introduite en 1683, mais il s'agit encore d'un plan réalisé a posteriori, après exécution. Dans la marine de guerre, le plan en trois vues (élévation ou plan longitudinal, sections horizontales, sections verticales) commence à précéder la construction vers 1720 , ce qui traduit un processus d'abstraction reposant sur une conception mathématique de la coque. À la fin du XviII siècle, un concours est lancé pour déterminer le meilleur plan pour chacun des rangs des bâtiments de guerre, réduits à trois $^{4}$ : des plans types, à partir duquel tous les navires de la flotte doivent être construits, sont sélectionnés. Cette évolution vers la standardisation va de pair avec une rationalisation de la formation des constructeurs. Dans la marine militaire, les maîtres-charpentiers sont progressivement remplacés, au milieu $\mathrm{du} \mathrm{xvIII}^{\mathrm{e}}$ siècle, par des ingénieursconstructeurs dont le corps est constitué par l'ordonnance du 25 mars 1765 . Les élèves doivent fréquenter l'École de Paris ${ }^{5}$, fondée par Henri Louis Duhamel du Monceau, après avoir été sélectionnés dans les ports et y avoir subi un apprentissage auprès de constructeurs en fonc- tion. L'institution d'un concours, en 1786, permet de recruter des élèves en dehors des ports et d'exiger d'eux des connaissances préalables en mathématiques, jugées plus utiles que l'apprentissage. Les ingénieurs-constructeurs deviennent, en 1800, les officiers militaires du génie maritime. Duhamel du Monceau fait don au roi et dépose au Louvre, en 1748, sa collection de modèles de navires; elle est d'abord exposée à la bibliothèque royale, puis à proximité de la salle des séances de l'Académie des sciences qui consacre au cours du $\mathrm{XVIII}^{\mathrm{e}}$ siècle de nombreux prix au perfectionnement du navire et contribue aux progrès de la navigation; mais la collection de modèles doit surtout servir de support à l'école des élèves ingénieursconstructeurs fondée par Duhamel du Monceau.

Dans la tourmente révolutionnaire, les académies et l'école des ingénieursconstructeurs quittent le Louvre, mais les modèles demeurent malgré les tentatives d'éviction de la part du nouveau Muséum des arts; ils sont cependant laissés à l'abandon et se détériorent rapidement. Les biens des émigrés touchant à l'art naval sont répartis entre la Bibliothèque nationale et le dépôt des cartes et plans de la Marine, où ils incarnent les progrès de la science géographique, tandis que des collections de modèles sont rassemblées dans un éphémère musée naval au ministère de la Marine et au palais de Trianon en tant que symboles de la puissance navale de la France. La fonction originale de support technique et pédagogique est perdue. Malgré ces efforts de mise en 
scène, l'image de la marine est ternie par les défaites de l'Empire, puis par les tentatives maladroites de la Restauration pour effacer les traces de la Révolution et de l'Empire : flotte réduite, réformes structurelles, réintégration des officiers émigrés qui retrouvent des commandements après être restés étrangers à la mer pendant vingt ans.

En 1818, le ministre de la Marine propose la renaissance de la salle de marine de Duhamel du Monceau sous le nom de "musée ", mais sa création n'est ratifiée que près de dix ans plus tard, en décembre 1827, quand la marine a retrouvé une partie de son prestige grâce aux efforts réformateurs et budgétaires menés par les ministres Molé et Portal et à la reprise des grands voyages d'exploration. Le projet de musée Naval, placé sous le patronage du Dauphin de France,
" [Contenir] les modèles des bâtiments de guerre de tout rang, et, autant que possible, ceux des navires de commerce de diverses grandeurs; les plans en relief des ports et de nos principaux établissements maritimes; les apparaux et machines spécialement en usage dans les arsenaux; les instruments employés pour la navigation, et les produits curieux des contrées nouvellement découvertes. À cette collection déjà fort intéressante par elle-même, se joindront les statues de nos marins les plus célèbres, ainsi que les tableaux destinés à conserver le souvenir des combats honorables pour le pavillon français ${ }^{6}$. »
Au-delà du prestige de la seule marine de guerre et de l'héroïsme des combats navals, le musée doit réconcilier la capitale avec le littoral et constituer une mémoire maritime au sens large : hommes, infrastructures, géographie et sciences nautiques sont à l'honneur. Héritier de la galerie de modèles de Duhamel du Monceau, il s'apparente à un arsenal en miniature et se trouve donc logiquement placé sous la responsabilité d'un ingénieur du Génie maritime. Le musée est d'ailleurs doté d'un atelier pour la restauration des modèles anciens et la construction de nouvelles maquettes. Au même moment, des salles de modèles ouvrent au public dans les ports, notamment sous l'impulsion de Charles Dupin, ingénieur du génie maritime et membre de l'Académie des sciences. La maquette y est investie d'une double fonction d'instruction et de récréation car il s'agit à la fois de former les spécialistes et de familiariser le public avec la construction navale. 


\section{Constitution des collections: I'empreinte des conservateurs}

Le premier conservateur du musée naval du Louvre, l'ingénieur Pierre Zédé, est envoyé dans les ports afin de collecter modèles et objets; il rassemble également au Louvre des collections provenant du ministère de la Marine et $\mathrm{du}$ palais de Trianon. Son successeur, Apollinaire Lebas, membre de l'Institut, considère que le musée doit être une « encyclopédie de marine en relief [qui] ne doit pas offrir seulement l'image complète de ce qui se fait aujourd'hui, elle doit reproduire au moins une idée de ce qui se faisait autrefois, en présentant la série de perfectionnements qui sont réservés aux travaux à venir. Sous ce point de vue, le musée naval est un véritable conservatoire ${ }^{7}$ ». Cette affirmation de Lebas invite à la comparaison avec le Conservatoire des arts et métiers où œuvrent également des ingénieurs du génie maritime : Dupin, qui crée la salle de marine du port de Toulon, enseigne au Conservatoire et l'on note chez lui comme chez Lebas une volonté de vulgariser et diffuser la science navale afin de former des ouvriers compétents. Le rôle joué par ces technocrates ${ }^{8}$ issus des premières générations de polytechniciens est prépondérant : ils sont les héritiers des ingénieurs-constructeurs $\mathrm{du}$ siècle précédent et d'une tradition de construction navale guidée par la science mais encore attachée aux gestes et aux opérations techniques. Ce sont à la fois des savants, des hommes de la technique et des hommes de pouvoir; certains mènent depuis les directions du ministère de la Marine une politique ambitieuse de constructions navales et d'aménagements portuaires malgré l'instabilité politique.

Au début des années 1830, l'atelier du musée compte onze ouvriers, mais bientôt la moitié est renvoyée dans les ports, ce qui porte un premier coup à la constitution de la flotte miniature puisqu'il faut dès lors faire transporter les modèles des arsenaux vers Paris. L'actualité militaire est à cette époque relativement anecdotique et consiste en quelques sièges et engagements ponctuels, essentiellement en appui de l'armée de terre, mais la marine de la Restauration et de la Monarchie de Juillet a renoué avec la tradition des voyages d'exploration. Le musée est tout naturellement destinataire des objets " exotiques " rapportés par les circumnavigateurs célèbres tels que Freycinet, Duperrey ou Dumont d'Urville; ils prennent place aux côtés des trophées de Bougainville et des premières reliques de l'expédition Lapérouse retrouvées à Vanikoro. Les officiers de marine sont invités à collecter des curiosités destinées à enrichir cette " sorte d'actualité vivante des découvertes maritimes ${ }^{9}$ ", tandis que la technique passe au second plan. Dans la première moitié $\mathrm{du} \mathrm{XIX}^{\mathrm{e}}$ siècle, les plans-types de Borda et Sané ne sont pas remis en cause, la flotte à voiles est performante et la France, assise sur ses acquis, hésite à prendre le tournant de la propulsion à vapeur. Le modèle, un temps considéré comme objet technique support de formation, redevient progressivement simple décor ou symbole 
de la puissance de l'État et le musée une vitrine et un instrument de promotion d'une arme et d'une politique.

En 1848, le musée Naval quitte le giron de la Marine pour passer sous l'administration des Beaux-Arts; il devient un département du Louvre, au même titre que la peinture, la sculpture ou les antiquités égyptiennes. Ce changement de tutelle modifie sensiblement les conditions de fonctionnement du musée. Les ouvriers de l'atelier sont rappelés dans les ports et le coût de transport des modèles qui continuent à être produits dans les arsenaux est désormais à la charge de l'administration des musées. L'indigence des budgets d'acquisition et de fonctionnement devient un problème qui sera récurrent tout au long du siècle. La rupture avec la Marine est consommée avec la nomination, en 1852, au poste de conservateur de l'artiste Antoine-Léon Morel-Fatio, même s'il porte le titre, honorifique, de peintre officiel de la Marine : l'organisation initiale reposant sur une administration par un ingénieur $\mathrm{du}$ génie maritime est définitivement abandonnée. L'institution reçoit de toute façon bien plus de spécimens transportés par les navires de la marine royale que de modèles produits par ses arsenaux : collections ethnographiques, échantillons rapportés par la mission de Chine donnés par le ministère du Commerce ${ }^{10}$, etc. En gestionnaire avisé, Morel-Fatio propose la création d'une annexe ethnographique qui doit permettre au musée de Marine de se recentrer sur ses attributs légitimes : c'est cette composante " annexe » du musée qui mobilise l'essentiel de son attention et concentre la majorité des entrées sous le Second Empire. Seules les expositions univer- selles permettent de maintenir les collections de modèles à jour des innovations techniques; toutefois, les collections de marine ne s'accroissent plus guère. Le conservatoire des musées nationaux, instance de gouvernance du Louvre, profite de la mort de Morel-Fatio, en 1871, pour tenter de se débarrasser de son département naval, mais les circonstances politiques et économiques n'autorisent pas le transfert rapide des collections voulu par les Beaux-Arts. Le musée passe alors sous la direction d'un officier de marine qui est aussi artiste et homme de technique et, surtout, de nouveau un homme de pouvoir. L'amiral Pâris est membre de l'Académie des sciences, du Bureau des longitudes, de la Société de géographie, ce qui lui assure un réseau étendu en même temps qu'il conserve de solides appuis dans la Marine. Il donne un nouveau souffle au musée en suscitant et en sollicitant des dons, mais surtout en réactivant l'atelier qu'il finance en grande partie sur son salaire de conservateur. Le musée hébergeait jusqu'alors des collections hétéroclites, reflets de la confusion entre l'objet et sa fonction. Pâris recentre résolument la politique d'acquisition pour en faire un musée des techniques, un conservatoire de l'art naval et une encyclopédie de marine en relief, conformément au souhait de ses premiers conservateurs-ingénieurs. Il réintroduit la fonction documentaire et historique voulue par Lebas, afin d'en faire un lieu de mémoire de la technique nautique et un support à un enseignement qui, à la différence du Conservatoire des arts et métiers, lui reste étranger. L'école du Génie maritime n'entretient guère de relation avec le musée tandis que l'École $\mathrm{du}$ Louvre, ouverte à cette époque, ne 
propose évidemment pas de cours sur l'art naval. Pâris porte néanmoins une véritable vision technique. Il incarne le musée et sa personnalité contribue grandement au succès que les collections de marine rencontrent auprès du « grand public ».

Un autre officier de marine, l'amiral Miot, succède à Pâris en 1893. Il concentre son activité sur le réaménagement des collections, rendu nécessaire par l'accumulation d'objets dans des espaces de plus en plus saturés. Miot décède en 1900 et est brièvement remplacé par un architecte naval, Henri Guidicelli, à qui succède, en 1901, Jean Destrem, homme de lettres et journaliste clairement nommé pour débarrasser le Louvre du musée de Marine. Destrem a néanmoins laissé sa marque sur le musée : plus proche du « profil » de conservateur que ses prédécesseurs, il œuvre au récolement et à l'inventaire des collections et ne satisfait aucunement au souhait des détracteurs du musée. C'est sous sa direction, en 1905, que le musée ethnographique est dissous et ses collections dispersées ${ }^{11}$, ce qui contribue à renforcer l'unité et la singularité des collections de marine. Les projets de déménagement se succèdent : nombreuses sont les voix, adeptes comme détractrices, qui s'élèvent pour réclamer la disparition du musée de Marine du Louvre et prôner son transfert vers les Invalides, où il pourrait rejoindre le musée de l'Armée, ou vers tout autre écrin où il pourrait gagner en espace et en visibilité. Les surfaces allouées au musée sont en effet progressivement rognées et les jours d'ouverture au public se réduisent comme peau de chagrin, limités aux jeudis et dimanches entre 1905 et $1926^{12}$. Georges Clerc-Rampal, attaché au musée, consacre une salle à la guerre navale entre 1914 et 1918... au musée des Invalides, faute de place au Louvre. Les débats qui agitent le musée et l'opinion au début $\mathrm{du} \mathrm{xx}^{\mathrm{e}}$ siècle soulèvent parfois violemment la question, latente depuis un siècle, de la définition d'un musée de marine et de la nature de ses collections; ainsi, dans Navigazette, en 1911 :

"Que doit être le Musée de Marine? Une exposition rétrospective, recueil de souvenirs provenant de nos gloires maritimes, histoire documentaire, tangible, en quelque sorte, de l'évolution maritime depuis les temps reculés jusqu'à nos jours?

Il doit être cela, certes, puisque ce sont là les éléments qui le composent presque intégralement à l'heure actuelle. Mais accessoirement seulement. [...] L'archéologie navale est une science infiniment digne de la déférence des non-initiés, mais qui intéresse, il faut bien le dire, une toute petite sélection de savants distingués.

À côté de cela, le Musée de Marine [...] doit représenter avant tout un organe de vulgarisation maritime populaire. [...] En un mot, il doit être un instrument de propagande et d'éducation tout à la fois ${ }^{13}$. »

Les collections suffisent-elles à définir le musée ou le musée doit-il d'abord répondre à un projet? Un premier élément de réponse est apporté en 1919, quand le musée revient dans le giron du ministère de la Marine et devient musée de la Marine, puis est rattaché au Service historique de la marine qui rassemble archives et salles de modèles des ports. 
Les successeurs de Destrem sont des marins : le capitaine de frégate Edward Hubert et le capitaine de vaisseau Pierre Vincent-Bréchignac. Les conservateurs s'attachent à la valorisation de l'actualité navale à la faveur de la Première Guerre mondiale, mais l'état général des collections continue de se dégrader régulièrement, tandis que la tenue des inventaires laisse à désirer. Une proposition de transfert du musée dans la nouvelle aile du palais de Chaillot, émise en 1935, relance les débats et aboutit en pleine guerre, mettant fin à près de 150 ans de cohabitation entre collections de marine et de Beaux-Arts : le musée est inauguré en août 1943 dans les locaux qu'il occupe encore aujourd'hui.

\section{Les éléments d'un musée des techniques}

Lemusée de Marine produitses propres œuvres, dans la tradition technicienne et pédagogique du modèle de marine, mais il ne se réduit pas à ses collections de maquettes. Zédé, Lebas et Pâris ont joué un rôle important dans l'élargissement de la fonction du musée : centre de documentation et de conservation des plans, des outils, des matériaux, des modèles de navires à différents degrés d'achèvement, des représentations en situation. L'amiral conservateur a bénéficié d'une longévité qui lui a permis de marquer profondément le musée de son empreinte. Dès son arrivée au Louvre, il se positionne en effet dans la dynamique impulsée par Lebas, mais retombée du temps de Morel-Fatio, en se consacrant à la constitution de collections qui permettent enfin au musée de devenir un conservatoire de l'art naval. Plans, dessins, ouvrages et dossiers viennent compléter les modèles, peintures et sculptures, suffisants selon lui pour l'agrément du public mais non pour l'instruction des hommes de métier : écorchés et dioramas accentuent la dimension pédagogique tandis qu'une " bibliothèque purement technique ${ }^{14}$ " rassemble une documentation spécia- lisée. Destrem recense 912 ouvrages et 3753 pièces graphiques (aquarelles, estampes, photographies, dessins, plans, calques) dans la bibliothèque, provenant pour une grande partie de l'apport personnel de Pâris ${ }^{15}$. Pour ce dernier, le musée ne doit pas se contenter de montrer les modèles, il doit aussi conserver les sources fiables qui permettent aux historiens et aux archéologues de mener leurs recherches, aux constructeurs de s'inspirer des travaux de leurs prédécesseurs, aux marins de connaître dans ses moindres détails leur outil de travail. Les collections couvrent d'abord la construction navale, mais aussi d'autres réalisations industrielles liées à la navigation, telles que les phares, l'aménagement des ports, ou encore les grands travaux comme le percement du canal de Suez. Pâris déploie aussi une signalétique détaillée autour de l'œuvre, qui dépasse les simples cartels : cartes, notices longues, affiches portant des données numériques permettant la comparaison des modèles (dimensions, armement, équipage). Ces artifices documentaires apportent une indéniable valeur ajoutée aux collections. 
L'ambiguïté induite par la présence du musée de Marine au Louvre est balayée d'un revers de main par ses conservateurs successifs : d'une part, les collections renferment de "vraies » œuvres d'art (peintures, sculptures, objets représentant 760 numéros sur 2238 dans le catalogue Destrem), même parmi les éléments de navires (sculptures de poupe de la galère la Réale, figures de proues) (figure 1) ; d'autre part, les maquettes sont pour Pâris comme pour Destrem des modèles utiles aux artistes qui ne voient jamais les parties immergées des navires qu'ils représentent; le premier les considère même comme des chefsd'œuvre à part entière. Destrem apprécie la complémentarité des valeurs informative et artistique des collections : « Des chefs-d'œuvre il y en a; il y a des œuvres premières; il y en a d'honorables; il y en a aussi de médiocres que leur utilité documentaire a fait admettre ${ }^{16}$. » C'est cette polysémie qui distingue les collections de marine de celles de beaux-arts et tendrait à les rapprocher par certains côtés des collections d'arts décoratifs rassemblées à la faveur des expositions universelles. Le conservatoire des musées nationaux n'intervient d'ailleurs que très rarement dans les choix d'acquisition du musée de Marine.

Il est important d'insister sur l'ambiguïté du projet initial, à la fois conservatoire des techniques de construction navale et de navigation, musée de l'histoire navale et outil de propagande, le tout rassemblé au sein du Palais des beaux-arts. Le volet technique, essentiellement valorisé au $\mathrm{XIX}^{\mathrm{e}}$ siècle, reste en

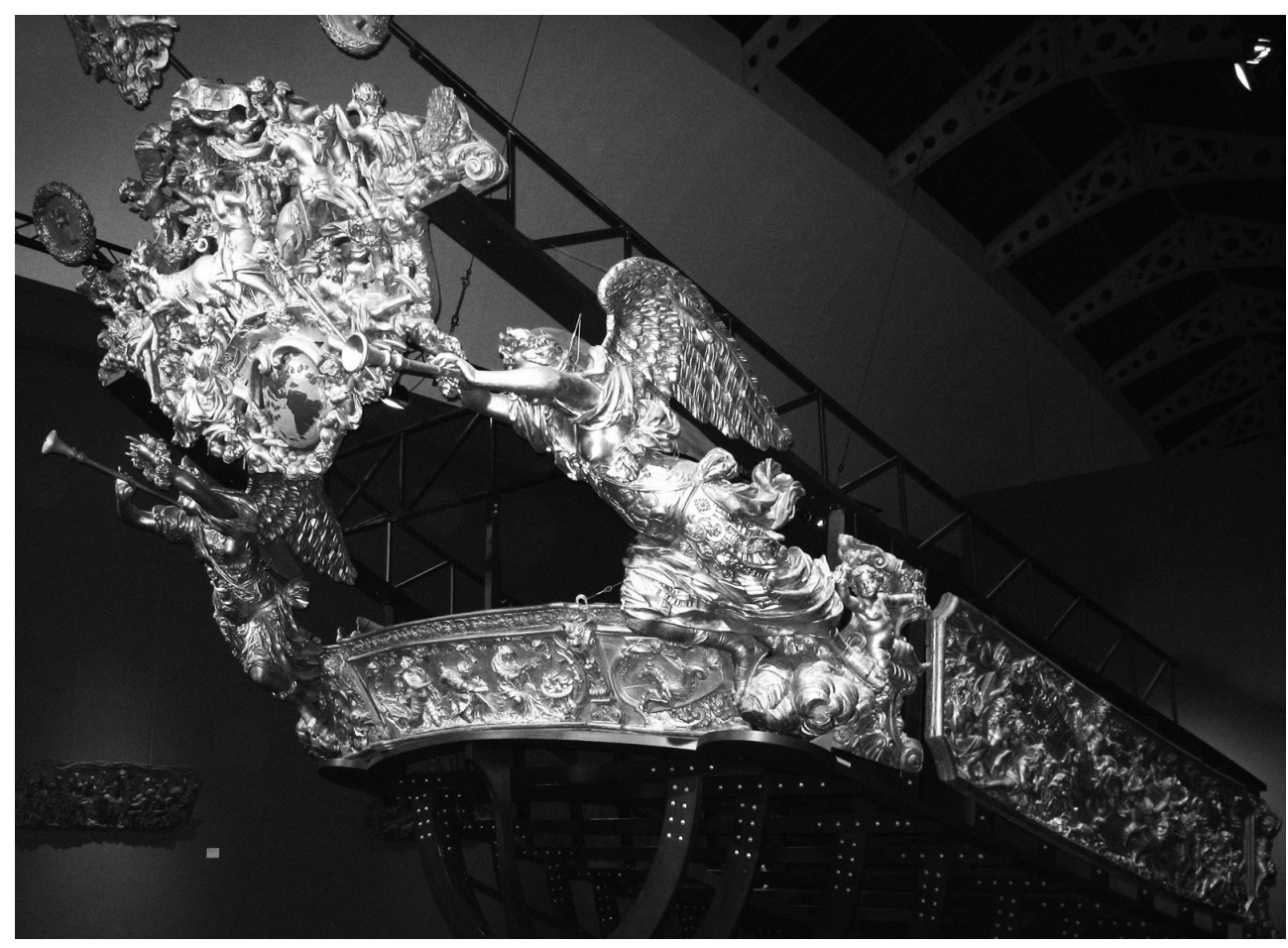

Fig. 1. - Poupe de la galère la Réale, Musée national de la Marine, Paris. Photo de l'auteur. 
effet déconnecté de son public, employé ou en formation en mer ou dans les ports. La mission de propagande semble avoir été mieux remplie auprès d'un public essentiellement familial et d'en- fants émerveillés par l'illustration de leurs rêves d'explorations et d'aventures maritimes. Ces objectifs équivoques se retrouvent dans les choix de classement et de mise en espace des collections.

\section{La mise en espace, compromis entre contraintes spatiales et choix muséographiques}

Le musée de Marine est d'abord installé au premier étage de la cour carrée du Louvre, dans l'aile donnant sur la rue de Rivoli. En 1838, il monte d'un étage et occupe quatorze petites salles en enfilade. Jusqu'à ce que Morel-Fatio crée l'annexe ethnographique, les salles ressemblent à un cabinet de curiosité : accumulation d'objets disposés selon des considérations esthétiques, faisceaux d'armes, compositions diverses, ensemble de souvenirs (figure 2).

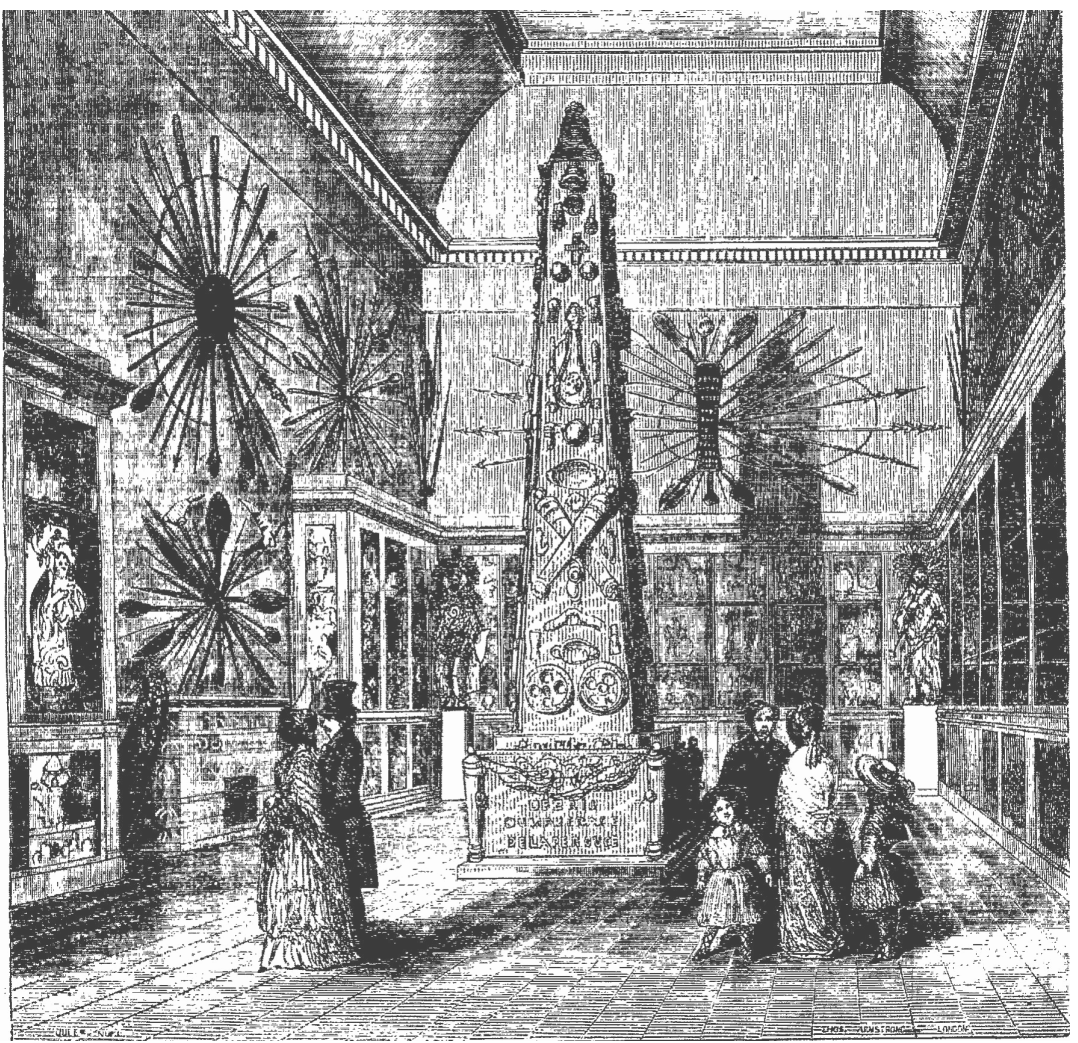

Fig. 2. - Le musée de Marine du Louvre, Magasin pittoresque, 1847. Photo de l'auteur. 
La création de l'annexe ethnographique permet d'aérer la mise en espace des collections de marine et d'envisager l'organisation de salles thématiques, mais les contraintes spatiales restent prépondérantes : les murs sont exploités pour disposer les tableaux, les vitrines doivent être placées au centre des pièces pour permettre la circulation des visiteurs. Il n'existe que très peu d'images des salles du musée et de la mise en espace des collections au Louvre. Les principales sources qui permettent de se faire une idée de l'agencement matériel des collections sont les demandes d'extensions et celles de mobilier adressées au conservatoire des musées ${ }^{17}$, et les guides touristiques qui présentent succinctement les différentes salles du Louvre. On constate alors une évolution sensible du cabinet de curiosité vers des espaces thématiques et une muséographie plus raisonnée. Aux belles armoires conçues spécialement pour le musée Dauphin par l'ébéniste Jacob-Desmalter, Pâris préfère substituer des vitrines de sa conception, sobres mais fonctionnelles. Les objets sont plus visibles, l'espace mieux exploité. Il récupère un couloir pour en faire la " galerie des pirogues ", dans laquelle il rassemble tous les modèles d'embarcations extra-européennes en leur adjoignant les armes, outils et autres objets provenant des «peuples sauvages". Il consacre une salle à Ferdinand de Lesseps : il y installe un plan-relief du canal, des panoramas de l'isthme, un buste de l'entrepreneur, des modèles de dragues, excavateurs et autres machines ayant servi au creusement du canal. Une salle est dédiée aux innovations les plus récentes : cuirassés, torpilles, phares. Mais la volonté d'ordonnancer la présentation ne résiste pas à l'envie de tout montrer : les salles sont vite saturées d'objets mais aussi de public, ce qui est funeste tant pour le bon déroulement de la visite que pour la sécurité des collections.

Miot donne, en 1898, une description de la disposition des salles ${ }^{18}$ (figure 3). Elle confirme que les tentatives de créer des salles thématiques sont vaines dans un espace si contraint; certaines juxtapositions confinent au bric-à-brac.

\section{Musée ethnographique \\ II. Musée ethnographique \\ III. Bâtiments de 1690 à 1715 \\ IV. Instruments de navigation et d'observation, tableau où sont répartis les différents modèles de bâtiments latins \\ V. Corderie, pouillerie, scierie, pompes}

VI. Spécimens de câbles

VII. Galères antiques et bâtiment scandinave retrouvé près de Christiania en 1888

VIII. Monument à la mémoire de Lapérouse, torpille Whitehead, modèles de feux de ports, bouées lumineuses, bateaux-feux, phares, tours, réduction de l'île de Vanikoro; puis salle des cuirassés IX. Canons, artillerie

\section{Artillerie}

XI. Spécimens des bâtiments du commerce, paquebots, gouvernails, ancres, cuisines, machines de ports et arsenaux

XII. Collection de modèles

XIII. Machines et obélisque de Louxor

XIV. Modèles des types de construction de 1798 à 1825 (collections 


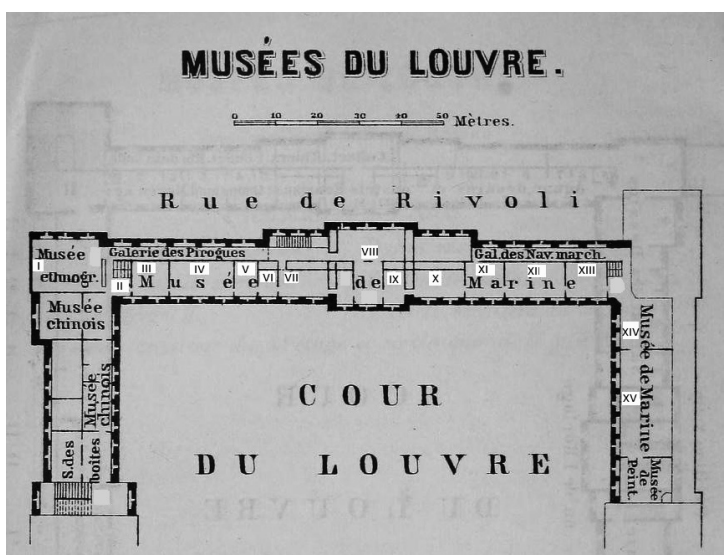

Fig. 3. - Plan du second étage du Louvre, d'après Karl Baedeker, Paris et ses environs, 1896.

de Trianon et du ministère de la Marine)

XV. Différents types de bâtiments de grande dimension ou de disposition spéciale, canal de Suez, abattage en carène de l'Artémise à Tahiti

Sans $n^{\circ} \quad$ Couloir des pirogues

Sans $n^{\circ} \quad$ Couloir des caboteurs (bateaux de pêche et caboteurs d'Europe)

Pendant la Première Guerre mondiale, des travaux permettent d'affecter une salle à la sculpture et aux décorations navales et objets-souvenirs, une encore aux ports et arsenaux. Les autres salles sont toujours plus ou moins dédiées à la construction navale par ordre chronologique : marine antique, marine ancienne, marine de Louis XIV, 1830-1848, marine nouvelle (cuirassés, torpilleurs, sousmarins), paquebots, cargos, grands voiliers et caboteurs, bâtiments exotiques, sauvetage, plaisance, navigation polaire, machinerie, instruments de navigation. La mise en espace reste malgré tout contrainte par la disposition des lieux. Destrem s'en excuse en expliquant que
" telle salle est haute, telle autre est basse; celle-ci est bien éclairée, cette autre l'est assez mal; des portes sont assez larges et autorisent le passage des grandes vitrines; d'autres portes sont étroites et ne s'ouvrent qu'aux petits dispositifs ${ }^{19}$ ». La localisation des objets ne reflète donc pas fidèlement l'organisation intellectuelle des collections et rend indispensable la rédaction de catalogues qui traduisent la conception à la fois technique et historique $\mathrm{du}$ fonds, soulignent ses richesses et ses lacunes. 


\section{Inventaires et catalogues}

La vie des collections de marine n'est pas un long fleuve tranquille, on l'aura compris. Les inventaires reflètent les strates de gestion qui se sont succédé. Dans son entreprise de recensement exhaustif des collections, Destrem identifie plusieurs numérotations successives correspondant à autant d'inventaires. Buache a réalisé l'inventaire de la collection Duhamel du Monceau en 1793 : cet inventaire, dit « ancien », est donc antérieur à la création du musée et recense 124 modèles, 22 livres et divers matériaux. Le premier inventaire du musée de Marine, établi en 1832 et dénommé par Destrem " inventaire Louis-Philippe ", compte 1272 numéros recensant pour un tiers les collections navales, pour les deux autres tiers la collection ethnographique $^{20}$. Morel-Fatio inaugure un nouvel inventaire qui sera poursuivi, mis à jour et annoté par ses successeurs. En 1853, les collections navales comportent 962 numéros et, en 1871, elles atteignent le $n^{\circ}$ 1052. L'accroissement s'accélère dans les premières décennies de la III République. Destrem annonce

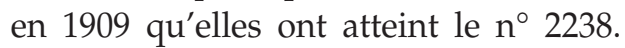
En 1919, on en est au n ${ }^{\circ} 2363$; Destrem signale en outre que la bibliothèque compte 1599 ouvrages et 5255 documents graphiques. Vingt-cinq ans plus tard, les collections de marine atteignent le $n^{\circ} 2773$, signe que la dynamique de croissance ne s'est pas enrayée malgré les aléas de la vie du musée.

Ces différentes strates de numéros $\mathrm{d}$ 'inventaires et de cotes se recoupent partiellement : le musée est un véritable puzzle archivistique, renforcé par la segmentation des fonds entre musée et docu- mentation et entre collections de Marine et fonds ethnographique. L'inventaire dit Morel-Fatio est néanmoins particulièrement riche d'enseignements sur la constitution des collections : y sont enregistrés le numéro d'inventaire, la date d'entrée dans les collections, une description de l'objet accompagné de la date de création, de l'échelle et de son origine (par exemple " modèle de... réalisé à partir du plan dessiné par... en $18 \ldots$ à l'échelle... »), le nom du modéliste pour les maquettes, le prix d'achat ou une estimation de la valeur, la provenance (achat par l'administration des musées ou don, avec mention du donateur). Les conservateurs successifs ont annoté ces registres pour y porter les nouveaux numéros d'inventaire ainsi que la correspondance avec le catalogue Destrem/ Clerc-Rampal (voir plus loin). L'étude des inventaires est donc indispensable pour la compréhension des modalités de constitution des collections. Il existe aussi des inventaires rétrospectifs par typologie de documents, à l'exemple de celui des plans et calques établi par Pâris qui décrit assez précisément le sujet du plan mais donne peu de détails sur l'objet lui-même : ce travail est plus celui d'un technicien que d'un conservateur.

L'analyse des catalogues apporte un éclairage différent sur les collections : les volumes d'accroissement des collections n'y sont pas identifiables, mais les conservateurs y proposent un cadre de classement intellectuel qui doit guider le visiteur dans sa découverte des objets exposés et compenser leur répartition hasardeuse dans les différentes salles. Morel-Fatio établit le premier catalogue 
du musée de Marine en $1853^{21}$ et finit le récolement des collections trois ans plus tard. C'est à lui que l'on doit la fixation de la première classification des objets de marine en quatre catégories :

1. Ports et arsenaux (plans-reliefs de ports, modèles de machines, d'engins, de sémaphores et phares, d'équipements de défense)

2. Constructions navales (échantillons de matières premières, modèles de bâtiments en construction, détails)

3. Modèles de bâtiments, catégorie subdivisée en marine ancienne (1696-1786) et marine moderne

4. Objets d'art et pièces historiques

Ce cadre de classement s'attache au processus de construction, de l'arsenal au navire achevé. L'ouvrage connaît un certain succès puisqu'il compte six tirages entre 1853 et $1872^{22}$ pour un total de 5000 exemplaires. Constatant cependant quelques années plus tard que le catalogue Morel-Fatio « ne se débitait pas, [Pâris l'a] remplacé avantageusement par des étiquettes détaillées $^{23}$ » qu'il juge plus à même de renseigner le visiteur qu'un catalogue dont l'organisation ne reflète pas le parcours de visite. Miot confirme ${ }^{24}$ que ces cartels sont suffisamment nombreux et détaillés pour que le visiteur puisse se passer de catalogue. Il faut attendre la nomination de Destrem et de son adjoint, Georges Clerc-Rampal, historien naval, pour que soit entrepris un récolement des collections et un travail d'envergure qui aboutit à la publication d'un nouveau catalogue en 1909.

Le choix du catalogue, du livret ou des simples cartels renvoie à la question de la démarche supposée du visiteur et de la fonction attribuée au musée (promenade, instruction, découverte, recherche). Destrem et Clerc-Rampal jugent que, même pour le grand public, " la visite d'un musée sans catalogue équivaut à la lecture d'une carte muette ${ }^{25}$ » et que c'est pour répondre à « la double ambition de renseigner le grand public et de fournir une documentation aux marins ainsi qu'aux érudits que préoccupent les matières de l'archéologie navale ${ }^{26}$ » que sont rédigés les catalogues des collections. Ils étendent le cadre de classement établi par Morel-Fatio ${ }^{27}$ sans le remettre en cause, suivant " l'ordre des opérations à faire pour créer une marine dans un pays où il n'existerait rien à ce point de vue ${ }^{28}$ ». Les conservateurs ajoutent en particulier une division consacrée à l'armement, choix révélateur de l'importance des mécanismes d'attaque et de défense dans la stratégie maritime de la III République; la division consacrée à la marine marchande regroupe les objets et modèles acquis à la faveur des expositions universelles et des expositions maritimes, mais aussi la collection de bateaux extra-européens constituée par l'amiral Pâris, unique au monde et qui est restée au musée lorsque les objets ethnographiques en ont été extraits. Au sein de chacune des sept divisions, le classement est chronologique afin de permettre d'aborder les collections de manière horizontale ou verticale. Ce cadre de classement traduit la dimension historienne qui s'est particulièrement développée au musée dans le dernier quart du XIX ${ }^{\mathrm{e}}$ siècle en parallèle des recherches des historiens du navire et des archéologues navals. Il fait ressortir les lacunes, en particulier sur l'époque médiévale. 


\section{1- Ports et arsenaux :}

- plans de ports, de bassins, de cales de constructions, de travaux hydrauliques

- modèles d'usines, d'ateliers, de machines et outils en usage à terre

- modèles d'engins et d'apparaux pour les manœuvres de force, les machines à mâter, les grues

- modèles de machines à draguer, de pontons et de citernes flottantes et tout ce qui a trait à l'amarrage des bâtiments

- pompes et apparaux de service d'incendie

- défense fixe et armement des côtes, batteries et torpilles fixes

- service des Ponts et Chaussées

\section{2- Constructions navales}

- échantillons de matières premières

- modèles de détails partiels et de systèmes divers de construction

- modèles de mâture, gréement et pouliage des bâtiments à voiles latines

- modèles de mâture, gréement et pouliage des bâtiments à traits carrés

- chaudières et machines

- cabestans, ancres, chaînes et câbles

- gouvernails et leurs apparaux

- modèles de bâtiments à différentes époques de leur construction

- distributions et installations intérieures

- cuisines, caisses à eau, fours, appareils de chauffage et matériel de couchage

- calfatage des bâtiments

- embarcations

3- Armement des navires* : tout ce qui sert à l'attaque et à la défense

- canons, torpilles automobiles, blindages

- armes portatives

4- Navigation* (positionnement et observations diverses)

- navigation estimée

- navigation observée (instruments d'observation astronomique, chronomètres)

- timonerie (appareils de signaux, de mesure des mouvements du navire et d'océanographie)

\section{5- Modèles de bâtiments de guerre complets}

5.1 marine à rames

- marine antique

- marine des galères (XVI ${ }^{\mathrm{e}}-\mathrm{XVIII}{ }^{\mathrm{e}}$ siècles)

5.2 marine à voiles

- règne de Louis XIII

- règne de Louis XIV

- règnes de Louis XV et Louis XVI, jusqu'à 1800

- $1800-1850$

5.3 marine à vapeur

- depuis l'origine jusqu'en 1860

- marine cuirassée depuis la Gloire 
6- Marine marchande ${ }^{*}$

- marine à voile

- marine à vapeur et moteurs mécaniques

- caboteurs et pêcheurs marine de plaisance

- bâtiments spéciaux (expéditions polaires, sauvetage)

- bâtiments exotiques, pirogues

- bâtiments fantaisistes

7- Objets d'art

- œuvres à caractère documentaire

- portraits, bustes de marins célèbres

- objets historiques et commémoratifs

Les catalogues permettent d'inventorier les collections, de les organiser intellectuellement autour de critères à la fois techniques et historiques, de les documenter. Chaque entrée comporte un numéro, un titre, une date qui n'est pas celle de réalisation du modèle mais, dans les cas où le modèle est postérieur à la réalisation de l'original, la date de construction de l'original. L'échelle est précisée quand elle est connue, de même que la provenance et l'artiste pour les œuvres d'art, mais non le modéliste pour les maquettes. Pour la plupart, les notices s'accompagnent d'un commentaire remettant l'objet en contexte. Le catalogue Destrem détaille particulièrement les caractéristiques des navires et se présente comme un guide de visite indispensable à la découverte d'un univers technique complexe.

\section{Valorisation des collections}

Pâris ne perçoit pas l'intérêt du catalogue auquel il préfère les cartels, mais, en complément de son travail de consolidation des collections techniques, il mène une activité éditoriale originale et ambitieuse qui conçoit les ouvrages à la fois comme des " produits dérivés » du musée et comme un moyen de conservation qui n'est pas sans annoncer les politiques de numérisation et le développement de visites virtuelles. Il complète ce travail éditorial par une série d'articles qui promeuvent autant la construction navale que le musée qui y est dédié.
La première entreprise est indissociable de la politique de constitution des collections de modèles menée par Pâris dès son arrivée au musée : il s'agit des Souvenirs de marine. Collection de plans ou dessins de navires et de bateaux anciens ou modernes, existants ou disparus avec les éléments numériques nécessaires à leur construction, comprenant six volumes publiés entre 1882 et 1908 chez Gauthier-Villars (figure 4). Ces 360 planches sont le fruit d'une immense collecte de sources de la construction navale à l'échelle internationale; la plupart des plans ont servi à 


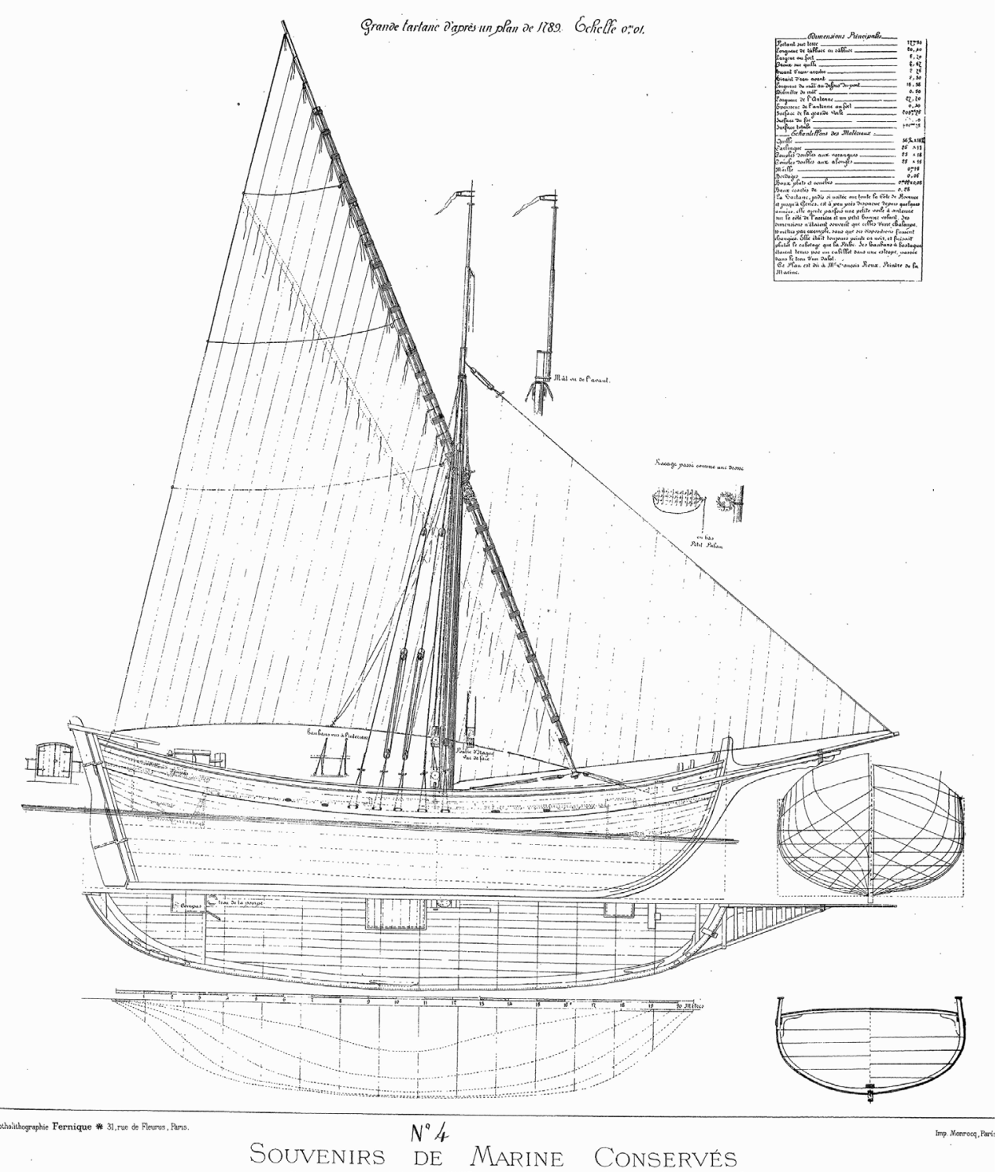

Fig. 4. - « Grande tartane d'après un plan de 1789 », planche des Souvenirs de marine conservés par Edmond Pâris. Source Wikimedia Commons.

réaliser les modèles en trois dimensions conservés au musée. Chaque planche contient tous les éléments numériques, textuels et graphiques qui doivent permettre de reproduire à l'identique les navires de tous les temps et de tous les pays.
En effet, à la différence des bâtiments de pierre, les navires ne se conservent pas au-delà de quelques décennies et il ne reste à l'archéologue et à l'historien que des plans et des modèles. Or ces modèles, eux aussi faits de bois et de ficelle, sont extrêmement fragiles; ils 
sont de surcroît soumis à l'humidité, à d'importants écarts de température ainsi qu'à l'affluence du public. Les plans originaux peuvent eux aussi se détériorer ou se perdre. Afin d'assurer une meilleure conservation de cette mémoire maritime, Pâris lance une campagne photographique pour fixer les objets par l'image et pouvoir les reproduire à l'infini. Le livre, de par sa reproduction et sa diffusion, constitue pour lui le plus sûr moyen de conservation en même temps qu'il est un support d'information et de vulgarisation. Le Musée de Marine du Louvre : histoire, description, construction, représentation, statistique des navires à rames et à voiles d'après des modèles et les dessins des galeries du Musée du Louvre (publié chez J. Rothschild en 1883) est à la fois un catalogue illustré, un livret de visite du musée et une encyclopédie historique de la construction navale. Il se compose de 153 pages de texte richement illustrées de gravures et de 60 planches «phototypiques inaltérables » reproduisant pour l'éternité des maquettes, gravures et tableaux conservés au musée.

Le successeur de Pâris, Miot, publie quant à lui une Promenade au musée de Marine $^{29}$ qui s'apparente aux notices des guides touristiques : description du musée salle par salle avec mise en relief des pièces maîtresses. Destrem rédige, en 1919, une histoire du musée ${ }^{30}$ qui paraît dans la Gazette des beaux-arts, et achève dix ans plus tard «Le musée de la Marine du Louvre, ses prédécesseurs, son histoire, les collections navales de nos arsenaux », publié à titre posthume en 1940; mais ces quelques textes semblent peu de choses au regard de l'entreprise éditoriale de Pâris dont les Souvenirs ont été réédités à plusieurs reprises dans le dernier tiers $\mathrm{du} \mathrm{xx}^{\mathrm{e}}$ siècle et consacrent la dimension technique que le conservateur du musée a véritablement incarnée pendant plus de vingt ans (1871-1893).

\section{Conclusion}

Malgré des aléas dus notamment à son rattachement aux Beaux-Arts, le musée de Marine s'est construit au long du XIX ${ }^{\mathrm{e}}$ siècle comme un musée des techniques spécialisé dans la construction navale. Cette dimension a bénéficié de quelques personnalités fortes attachées à l'architecture navale sous toutes ses facettes, soucieuses de patrimonialisation autant que d'innovation, de conservation et de diffusion, de formation et de vulgarisation. La situation au sein du Louvre constitue un handicap et brouille l'image du musée perçu d'abord comme celui d'une arme par les départements des Beaux-Arts, malgré les efforts consentis pour développer les collections consacrées à la marine de commerce, aux bateaux traditionnels du monde entier et aux nouvelles formes et vocations du navire (sauvetage, exploration, plaisance, sport). Le public semble davantage attiré par le fantasme d'aventure et d'exotisme véhiculé par les collections de marine que par une quelconque typologie des collections : les témoignages de visiteurs qui nous sont parvenus ${ }^{31}$ rendent compte d'un désir d'évasion satisfait par le pou- 
voir évocateur des objets exposés. Les navires en miniature semblent donner forme aux récits de voyage, aux romans maritimes en vogue au $\mathrm{XIX}^{\mathrm{e}}$ siècle aussi bien qu'à la littérature d'aventure ou à l'œuvre de Jules Verne. Le retour dans le giron de la Marine et le déménagement au Trocadéro au $x^{\mathrm{e}}$ siècle ne contribuent pas à clarifier l'identité des collections du musée employées essentiellement à des fins de propagande afin de magnifier les succès militaires de la Marine française, en particulier dans les périodes d'aprèsguerre. Les conservateurs continuent malgré tout de valoriser la diversité de ses collections. La nature technique des collections ne présage donc en rien de la volonté qui préside à leur rassemblement ni de l'interprétation que conservateurs et publics en dégagent. Au-delà des collections elles-mêmes, le lieu où elles sont exposées et le contexte dans lequel elles s'insèrent contribuent à leur donner sens et leur nature technique ne cesse d'être concurrencée par des enjeux à la fois identitaires, politiques et économiques ainsi qu'une projection des attentes d'un public qu'il s'agit de diversifier et de fidéliser. Les contributeurs de l'ouvrage Neptune au musée ont bien montré la multitude des approches et des muséographies possibles à partir de collections de modèles et d'objets de marine : histoire navale, espaces et circulations maritimes, gens de mer, métiers anciens et nouveaux liés à la mer, loisirs, etc. $C^{\prime}$ est à la définition d'un nouveau projet muséographique que s'attelle le musée national de la Marine aujourd'hui : une exposition rétrospective sur les soixantedix années passées au palais de Chaillot " inaugure " la fermeture du musée au printemps 2017 pour une durée de cinq ans. Le discours du ministre de la Défense annonçant ces grands travaux de rénovation témoigne de la tentation persistante de définir un projet protéiforme, entre rêve, pédagogie, patrimoine, propagande et aventure :

« Le nouveau musée sera un lieu vivant, interactif, porteur d'émotions et de savoirs; il invitera au voyage; il sera l'écrin d'un patrimoine exceptionnel, et les collections du musée seront valorisées comme jamais elles ne l'ont été; mais il sera aussi un outil puissant de sensibilisation aux enjeux de la mer aujourd'hui et demain. Plus que jamais, venir ici sera - au cœur de Paris - une manière de prendre la mer $^{32}$. »

Amener la mer à Paris reste donc le fil 161 directeur pour tailler ce joyau aux mille facettes.

\section{Notes}

1. Bertrand Daugeron et Armelle Le Goff (dir.), Penser - Classer - Administrer. Pour une histoire croisée des collections scientifiques, Paris, Publications scientifiques du Muséum national d'histoire naturelle - CTHS, 2014.

2. Patrick Louvier (dir.), Neptune au musée : puissance, identités et conflits dans les musées maritimes et navals, Rennes, Presses universitaires de Rennes (Art \& société), 2013.

3. Alain Niderlinder, « Le Musée de la Marine et ses collections. Éléments chronologiques ", Neptunia, no 193 à 197, 1994-1995.

4. Les rangs sont définis par rapport à l'armement : 74, 80 et 110 canons.

5. Dès avant la création du corps puisque l'école est fondée en 1741. Duhamel du Monceau est inspecteur général de la Marine et membre de l'Académie des sciences.

6. " Musée naval », Annales maritimes et coloniales, partie non officielle, vol. 1, 1828, p. 198-200. 
7. Archives nationales [ensuite AN], archives des musées nationaux, EM1, Apollinaire Lebas, « Note pour Monsieur le directeur des Musées nationaux », 25 juillet 1848.

8. Bruno Belhoste, La formation d'une technocratie : l'École polytechnique et ses élèves de la Révolution au Second Empire, Paris, Belin (Histoire de l'éducation), 2003; B. Belhoste souligne la progressive prise de pouvoir des corps savants issus de l'École polytechnique dans l'élite sociale et dans la gouvernance du pays au XIX ${ }^{\mathrm{e}}$ siècle.

9. Sylviane Jacouemin, Rao-Polynésies : exposition... au Musée des arts d'Afrique et d'Océanie, Paris, octobre 1992-mars 1993, Paris, Marseille, Éditions Parenthèses, Réunion des Musées nationaux (Arts témoins), 1992, p. 20.

10. Voir dans ce numéro Christiane Demeulenaere-Douyère, « Missions commerciales et collections techniques au XIX siècle : l'introuvable "musée chinois" de la mission de Chine ».

11. Entre autres, entre le musée d'ethnographie du Trocadéro, le musée des Antiquités nationales de Saint-Germain-en-Laye, le musée de La Rochelle. Il faudra attendre plus de vingt ans pour que le transfert des collections soit effectif.

12. Les salles sont même totalement fermées entre 1914 et 1919.

13. Maurice Rondet-SAInt, « Musée de Marine ", Navigazette, no 1133, 12 janvier 1911, p. 3-4.

14. Edmond PÂRIs, "Le Musée de marine ", Revue maritime et coloniale, vol. 34, 1872, p. 976-977.

15. Jean Destrem et Georges Clerc-Rampal, Catalogue raisonné du Musée de la Marine, Paris, Imprimerie française, 1909.

16. Jean Destrem, « Le musée de Marine du Louvre ", Gazette des beaux-arts, vol. 15, 1919, p. 382.
17. AN, archives des musées nationaux, série EM.

18. Paul-Émile Miot, Promenade au Musée de la Marine, Paris, Librairies-Imprimeries réunies, 1898.

19. J. Destrem et G. Clerc-Rampal, Catalogue raisonné du Musée de la Marine, op. cit., p. 396.

20. Alain NiderLinder, « Le Musée de la Marine et ses collections. Éléments chronologiques », Neptunia, no 195, 1994, p. 41-50.

21. Léon Morel-Fatio, Notice des collections du Musée de Marine exposées dans les galeries du Musée impérial du Louvre. $1^{r e}$ partie : Musée naval, Paris, Vinchon, 1853; les collections ethnographiques devaient faire l'objet d'une seconde partie qui n'a jamais vu le jour.

22. $1853,1855,1858,1860,1865,1872 \mathrm{chez}$ Vinchon puis Charles de Mourgues, imprimeurs des musées nationaux.

23. AN, F/21/4483, lettre de Pâris au ministre de l'Intérieur (au directeur des musées nationaux?), s. d.

24. In P.-É. Мiot., Promenade au Musée de la Marine, op. cit.

25. J. Destrem et G. Clerc-Rampal, Catalogue raisonné du Musée de la Marine, op. cit., p. xii.

26. Ibid.

27. Les divisions marquées d'un astérisque sont celles qui ne figuraient pas dans le classement proposé par Morel-Fatio.

28. J. Destrem et G. Clerc-Rampal, Catalogue raisonné du Musée de la Marine, op. cit.

29. P.-É. Мiot, Promenade au Musée de la Marine, op. cit.

30. J. Destrem, « Le musée de Marine du Louvre ", art. cit.

31. Articles de revues, et aussi poèmes et récits littéraires.

32. Discours de Jean-Yves Le Drian, 6 octobre 2015. 